JOURNAL OF SYNCHROTRON RADIATION

ISSN 1600-5775

Received 18 July 2017

Accepted 23 February 2018

Edited by G. Grübel, HASYLAB at DESY, Germany

Keywords: white-beam detector; fast imaging; phase contrast; scintillators; X-ray exposure; inert gas.

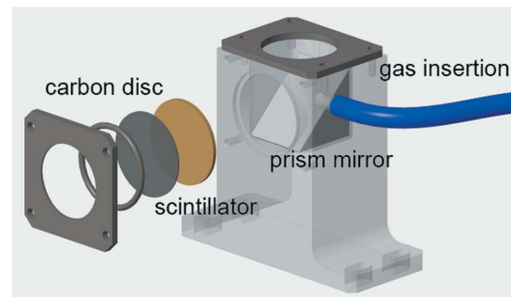

OPEN $\odot$ ACCESS

\section{Development of an X-ray imaging system to prevent scintillator degradation for white synchrotron radiation}

\author{
Tunhe Zhou, Hongchang Wang,* Thomas Connolley, Steward Scott, \\ Nick Baker and Kawal Sawhney
}

Diamond Light Source Ltd, Harwell Science and Innovation Campus, Didcot OX11 ODE, UK. *Correspondence e-mail: hongchang.wang@diamond.ac.uk

The high flux of the white X-ray beams from third-generation synchrotron light sources can significantly benefit the development of high-speed X-ray imaging, but can also bring technical challenges to existing X-ray imaging systems. One prevalent problem is that the image quality deteriorates because of dust particles accumulating on the scintillator screen during exposure to intense X-ray radiation. Here, this problem has been solved by embedding the scintillator in a flowing inert-gas environment. It is also shown that the detector maintains the quality of the captured images even after days of X-ray exposure. This modification is cost-efficient and easy to implement. Representative examples of applications using the X-ray imaging system are also provided, including fast tomography and multimodal phase-contrast imaging for biomedical and geological samples.

\section{Introduction}

Third-generation synchrotron sources generate hard X-rays with significantly higher flux than laboratory X-ray sources. Nevertheless, with monochromatic X-rays selected by crystal monochromators, only a small portion of the flux can be used, and the lowered flux inevitably limits the data acquisition speed. For many X-ray imaging applications, the requirement for temporal coherence is moderate, in which case the flux can be significantly increased by using multilayer monochromators with a larger spectral bandpass. However, the image quality often suffers from the irregular stripe modulations caused by imperfections in the multilayers (Rack et al., 2010), and the stripes are difficult to remove by flat-field correction in postprocessing. To maximize the usage of the high flux of the synchrotron radiation and further decrease the data acquisition time, a pink (with filters) or white beam can be used by removing the monochromator and other X-ray optics. In addition, image artefacts caused by imperfections in the beamline optics can also be avoided by using a pink or white beam. Taking advantage of such a high-flux beam, timeresolved X-ray imaging opened many new avenues of scientific endeavor. Examples include studies of fluid dynamics by $\mathrm{X}$-ray imaging, whereby the inaccuracy from the refraction and reflection in visible light photography can be avoided (Lee et al., 2013); imaging the dynamic behavior under extreme conditions of materials that are nontransparent in visible light (Eakins \& Chapman, 2014; Luo et al., 2012; Workman et al., 2010); and four-dimensional imaging with high-speed X-ray tomography revealing the dynamics of the internal three- 
dimensional structure of the samples (Walker et al., 2014; Baker et al., 2012).

To further enhance the image contrast and retrieve complementary information to absorption-contrast imaging, diverse $\mathrm{X}$-ray phase-contrast imaging techniques have been developed over the last two decades. Importantly, several of the phase-contrast imaging techniques, such as the propagation-based, the grating-based and the speckle-based methods, are compatible with a polychromatic beam and can therefore benefit from the high flux of white beam radiation (Wilkins $e t$ al., 1996; Momose et al., 2009; Wang, Kashyap, Cai et al., 2016).

When using a white beam, current X-ray imaging systems face several challenges. The high flux and high energy of the beam can easily damage the scintillator, objective and other instruments in the beam path through excessive heating or radiation damage. The scintillator screen can become electrostatically charged because of the intense ionizing radiation. As a result, dust particles are attracted to the screen, degrading the image in a non-systematic manner and in some cases appearing as over-saturated spots which cannot be flatfield corrected. Scattered high-energy photons directly striking the camera sensor can also lead to saturated white spots (commonly known as 'zingers'). An X-ray imaging system designed in order to overcome some of these problems is presented in this paper. We hope it will benefit other research groups in the community facing similar challenges. Experimental results from a tomographic scan and from a scan using the speckle-based phase-contrast technique are presented as demonstrations of potential applications of the $\mathrm{X}$-ray imaging system.

\section{Experimental setup and detector design}

Tests of the detector were conducted at the bending magnet (BM) beamline B16 (Sawhney et al., 2010) at Diamond Light
Source (Diamond). The BM source produces a continuous spectrum of X-rays with a critical energy of $8.4 \mathrm{keV}$, with the spectrum extending to beyond $100 \mathrm{keV}$ on the high-energy side. The experimental layout is illustrated in Fig. 1. Raw emission, with no narrowband spectral filtering from monochromators or focusing optics, was used. A $0.5 \mathrm{~mm}$-thick aluminium attenuator was used to protect the downstream components in the beam path, such as beryllium and Kapton windows, from excessive radiation. Several beam slits were placed at various positions along the beam path to reduce the stray light and heat load on components placed upstream of the camera. Filters with different thicknesses and materials could be moved into the beam, with motorized stages prior to the sample, to tune the peak energy of the broadband beam. Spectra simulated with the $X$-ray Oriented Program (XOP) (Sanchez del Rio \& Dejus, 2004), using aluminium and copper filters, are given as examples of this approach in Fig. 1. The simulation shows that the flux would be dramatically reduced by increasing the thickness of the copper filter, and the peak energy of the spectra would shift towards a higher energy region. Pre-hardened X-rays may be preferred, especially for samples that are thick or contain high-atomic-number materials, as the low-energy photons only increase the absorbed dose in the samples yet contribute little to achieving image contrast. The BM source was $41 \mathrm{~m}$ from the beam slit, and $47.5 \mathrm{~m}$ from the detector, between which the sample to be imaged could be placed flexibly. The camera and optics housing were lined with a lead shield protecting them from scattered X-rays and to reduce the bright pixels caused by scattered high-energy photons.

The design of the detector (Fig. 1) follows the concept of the indirect detector approach (Bonse et al., 1991; Stampanoni et al., 2002; Koch et al., 1998), with the scintillator imaged by a CMOS camera via a lens. The camera and the lens are each independently mounted on a linear stage. By adjusting the

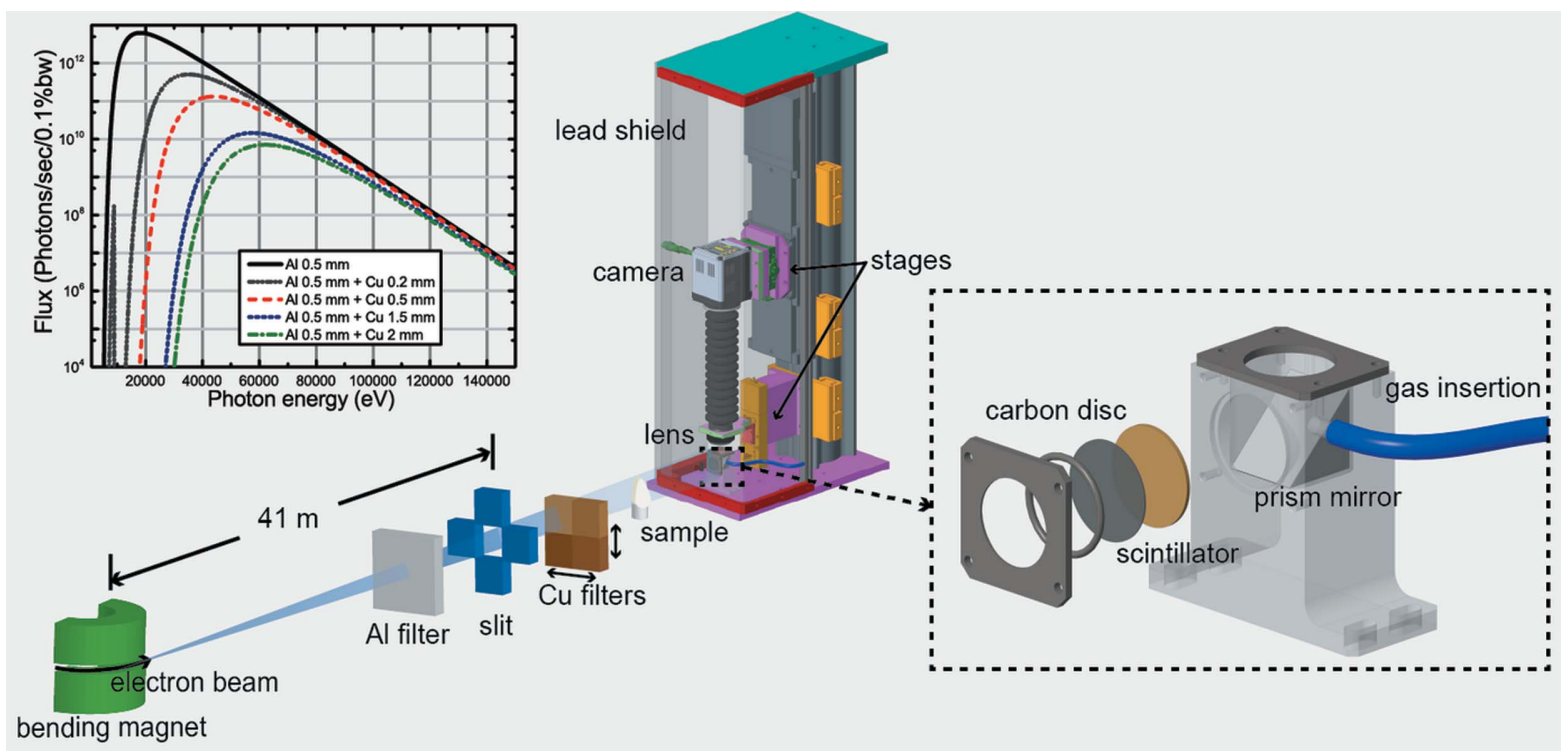

Figure 1

Illustration of the beamline imaging setup and camera (not to scale), and simulated spectra for a BM source at Diamond with different filters. 

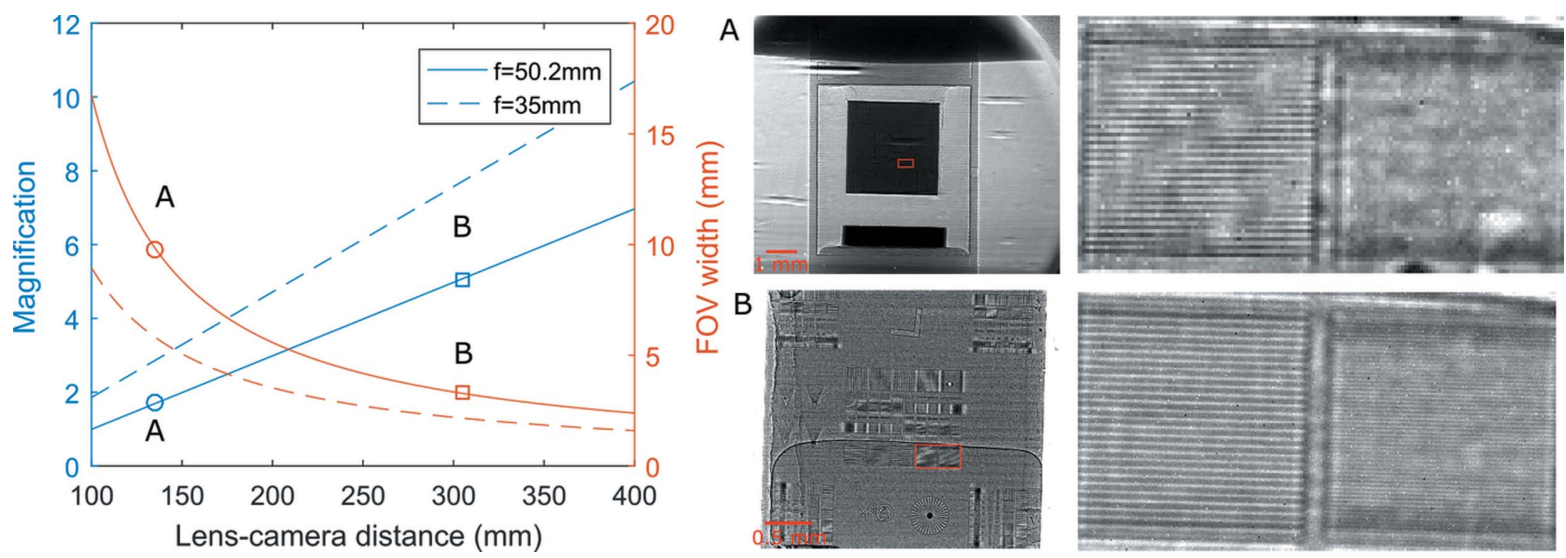

Figure 2

Theoretical calculations of magnifications and corresponding widths of FOV for different distances between the lens and the camera for two lenses. Images of a resolution sample taken at positions A and B are given, showing the line patterns with widths of 4 and $2 \mu \mathrm{m}$.

positions of the lens and the camera, the geometric magnification can be varied continuously without the need to change to objectives with different magnifications. An additional goniometric stage is mounted in order to align the orientation of the camera sensor to the rotation axis of the sample stage for tomographic scans. With the current lens $(50 \mathrm{~mm} f / 2.8$ Schneider macro lens) and motion-stages $(150 \mathrm{~mm}$ and $25 \mathrm{~mm}$ travel range), the magnification can be varied between 1.7 and 5.1, shown as positions A and B in Fig. 2. Two radiographic images of a resolution sample (Micro-CT Bar Pattern NANO) at these two different geometrical magnifications are also given in Fig. 2. The patterns with linewidths of 4 and $2 \mu \mathrm{m}$ are shown in the images, where we can see that the finer line pattern with a linewidth of $2 \mu \mathrm{m}$ is at the limit of the resolution with the higher magnification setting. If a higher magnification is desired with the same overall camera dimensions, then a macro lens with shorter focal length can be used, as shown by the dashed lines in the plot in Fig. 2. Compared with microscope objectives with relatively small magnifications, a macro lens has a larger numerical aperture, and hence higher collection efficiency. Long-working-distance microscope objectives with higher magnifications are good alternatives for applications that require even higher spatial resolution. The pco.edge camera has a pixel size of $6.5 \mu \mathrm{m}$ and $2560 \times 2160$ pixels. The field of view (FOV) and effective pixel size changes along with the varying of magnification. This tradeoff between a larger FOV and higher spatial resolution is illustrated in the plot in Fig. 2. The maximum frame rate of the camera is 100 frames s $^{-1}$ at full resolution. With a reduction of the total pixel number by using only a region of the sensor, the frame rate can be increased. Depending on the application, existing cameras on the market can reach frame rates as high as $\mathrm{MHz}$ and capture even single-bunch X-ray images (Etoh et al., 2003; Olbinado et al., 2017), with a sacrifice of either FOV or spatial resolution.

The scintillator used was LuAG:Ce from Crytur with a thickness of $80 \mu \mathrm{m}$ and a diameter of $25 \mathrm{~mm}$. A glassy carbon window was mounted in front of the scintillator to block visible light and minimize contamination of the external surface by dust. Olbinado et al. have shown that LuAG:Ce is one of the scintillator materials whose emission spectra is best matched to CMOS sensor quantum efficiency (Olbinado et al., 2017). The thickness of the scintillator affects the spatial resolution and the conversion efficiency, which can be altered depending on the requirement of the experimental application. It should be noted that the scintillator can be replaced with others that have shorter decay times, such as LYSO:Ce or LuI3:Ce (Marton et al., 2014; Pidol et al., 2004), which are more suitable for ultrafast imaging.

The scintillator was mounted on a $45^{\circ}$ prism mirror cage, so that the lens and the camera could be mounted transverse to the X-ray beam direction allowing it to be protected from radiation damage caused by the $\mathrm{X}$-rays that passed through the scintillator. Dry nitrogen gas with a positive gas pressure constantly flowed into the mirror cage with a slow speed so that the dust from the surrounding environment could not enter the mirror cage and accumulate on the charged scintillator screen during X-ray exposures. Gas flow is easier to handle and more cost-efficient than a vacuum environment for the scintillator. As can be seen from the example given in Fig. 3(a), without any gas flow, a significant amount of dust accumulated after using the camera with a pink beam for $38 \mathrm{~h}$, which manifested as clouds of white spots in the X-ray image. The image quality was significantly degraded compared with that at the beginning of the test. In this case, the scintillator normally needed to be cleaned, or sometimes replaced. In Fig. 3(b), when the gas flow was on, the FOV was still clean after being used for $43 \mathrm{~h}$. This gave a much more stable image background for long duration or high-throughput experiments, thereby enabling a better comparison against time for applications that involve dynamically changing samples. The different efficiencies of individual scintillators and the slight variation in camera focus when manually switching the scintillators can also bring inconsistency into the collected images, which is best avoided for most applications. Eliminating the need to change and clean scintillators during experiments also offers benefits of reduced costs and downtime. 


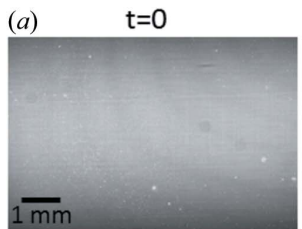

(b)

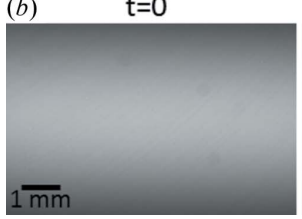

$9 \mathrm{~h}$

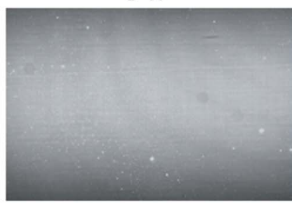

$8 \mathrm{~h}$

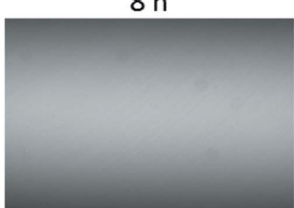

Figure 3

Flat-field image taken after scintillator is exposed with a pink beam $(a)$ without and $(b)$ with nitrogen gas flow.

\section{Applications}

\subsection{Fast imaging}

With the improved X-ray imaging system installed, we will be able to take full advantage of the much higher total flux of a white or pink beam compared with a monochromatic beam, and the required acquisition time can be significantly reduced. A more efficient use of beam time is therefore possible, as well as lessening the risk of artefacts from instabilities in fixation of sample or mechanical drifts during long exposures. This is especially valuable for experiments including tomographic scans and multi-dimensional raster scans. The high flux of the white beam and a camera that is compatible with such high radiation also makes it possible for fly scans (continuous rotation mode) (Kalender et al., 1990; Lak et al., 2008), or time-resolved experiments.

A tomographic scan was conducted at B16 during camera tests, and is shown in Fig. 4. Half of a human primary tooth was imaged with an exposure time of $50 \mathrm{~ms}$ for each projection with 601 projections taken over $180^{\circ}$. The effective pixel size was $3.8 \mu \mathrm{m}$. The beam was filtered by $0.5 \mathrm{~mm} \mathrm{Al}$ and $0.5 \mathrm{~mm} \mathrm{Cu}$, leading to a peak X-ray energy of $43 \mathrm{keV}$ as shown in Fig. 1. Fig. 4(a) shows a projection image from the scan. The image is flatfield and dark image corrected. The standard deviation of the background is 0.025 . The inset shows part of the dentine in the tooth, where giant tubules or microcanals can be observed (Agematsu et al., 1990, 2005; Sumikawa et al., 1999). The enamel, dentine and pulp of the tooth are clearly distinguishable, thanks to edge-enhance-

$26 \mathrm{~h}$

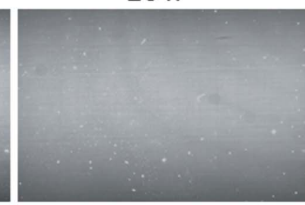

$23 \mathrm{~h}$

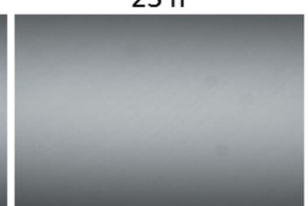

$38 \mathrm{~h}$

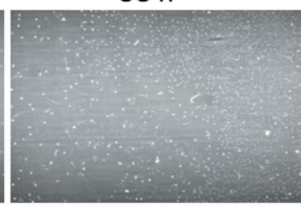

$43 \mathrm{~h}$

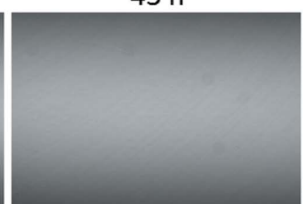

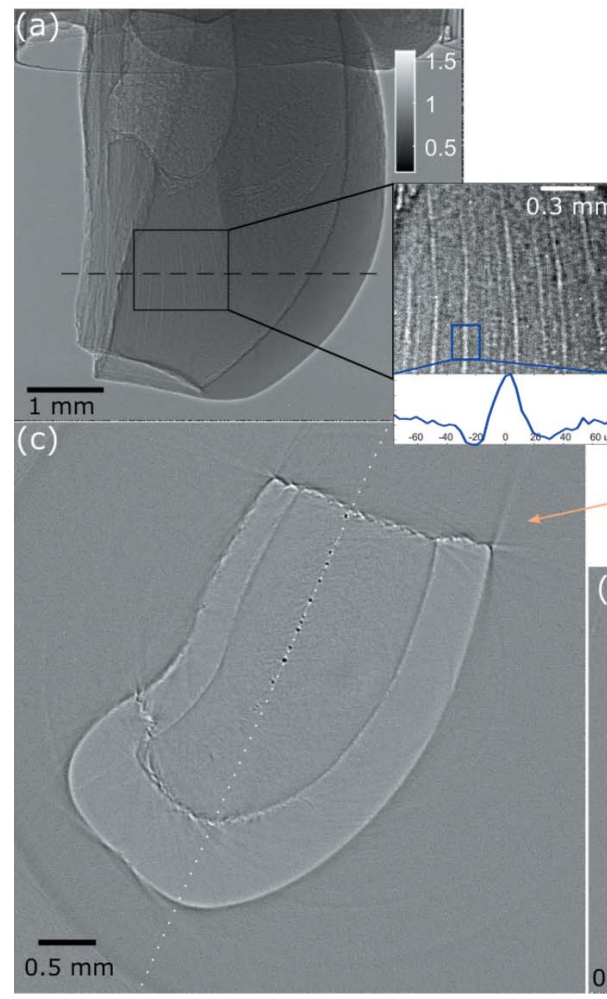

(b)

ments from phase contrast. Fig. 4(b) shows the three-dimensional rendering of the tomographic scan. Fig. 4(c) shows a transverse slice at the position indicated by the dashed line in Fig. 4(a), showing that the microcanals are aligned along the mesiodistal direction, in agreement with previous studies (Sumikawa et al., 1999; Agematsu et al., 2005). Fig. $4(d)$ is a vertical slice taken from the dotted line in Fig. 4(c), showing the pulp chamber and the propagating direction of the microcanals. From the inset with enhanced contrast, we can also see the structure of tubules in the dentine.

Previous studies on the microstructure of teeth have been mostly carried out using light microscopy or scanning electron microscopy (SEM). Both methods require the sample to be cut into thin slices, in order to observe the surface structure. Prior knowledge is required to find the region of interest for sample preparation. Drying and gold sputtering are also normally needed for SEM observations (Janda, 1995). X-rays, on the other hand, can image the internal three-dimensional structure non-destructively. The sample can be preserved in a more

\section{Figure 4}

(a) Projection image of half of a primary tooth with an exposure time of $50 \mathrm{~ms}$. The inset shows the dentine of the tooth with microcanals. (b) Three-dimensional rendering of the tomography. (c) Reconstructed transverse slice taken from the position indicated by the dashed line in $(a)$, revealing the microcanals aligned in the mesiodistal direction. $(d)$ Vertical slice taken from the dotted line in $(c)$ along the microcanals, with inset showing the tubules in the dentine. 
natural state to avoid artefacts and damage that can be introduced by the sample preparation process; therefore, more realistic information about the sample can be extracted. Fast and large-FOV tomography can also be applied as a pre-scan before the destructive sample preparation process for other imaging tools with higher resolution, such as SEM, or more recently, ptychography (Zanette, Enders et al., 2015), so that sample cutting can be controlled and targeted at the region of interest.

\subsection{High-energy phase-contrast imaging}

When the white-beam from the BM is filtered, low-energy photons are absorbed and a spectrum with a higher average energy is achieved. Materials that have high atomic numbers can be imaged and characterized, for example, with phasecontrast imaging methods (Spanne et al., 1999; Wang, Kashyap, Cai et al., 2016; Diemoz et al., 2016; Willner et al., 2013). With multimodal phase-contrast imaging, results about phase, absorption and scattering can be obtained simultaneously, which offers complementary information to the material properties compared with commonly used attenuation-contrast imaging (Pfeiffer et al., 2008; Berujon, Wang et al., 2012; Modregger et al., 2016; Pagot et al., 2003). In the example given here, the speckle-based phase-contrast technique with one-dimensional scan mode has been applied (Wang et al., 2015, 2016), where a stack of sandpaper (P800) is used as the static diffuser. An image of the sandpaper is given in Fig. 5(a), with the random structures resembling 'speckles'. The effective pixel size was $3.8 \mu \mathrm{m}$. In this experiment, $0.5 \mathrm{~mm}$-thick $\mathrm{Al}$ and $1.5 \mathrm{~mm}$-thick $\mathrm{Cu}$ filters were used, which gave a hardened spectrum with peak energy at $57.5 \mathrm{keV}$, as shown in Fig. 1 . In this case, the visibility of the speckle pattern, defined as the normalized standard deviation here (Zanette, Zdora et al., 2015), is about $7 \%$. The speckle size calculated as the FWHM of the autocorrelation is $12 \mu \mathrm{m}$. Other materials, such as steel wool (Wang, Kashyap, Cai et al., 2016), can also be used as diffuser, which can give a different visibility and speckle size.

The principle of the speckle-based phase-contrast imaging is to measure the refraction of the X-ray beam induced by the sample, by tracking the shift of the speckle pattern. With the proportional relation $(\partial \Phi / \partial x, \partial \Phi / \partial y)=$ $(2 \pi / \lambda)\left(\alpha_{x}, \alpha_{y}\right)$, where $(\partial \Phi / \partial x)$ and $(\partial \Phi / \partial y)$ are the phase gradients in the horizontal and vertical, respectively, $\alpha_{x}$ and $\alpha_{y}$ are the refraction angles and $\lambda$ is the wavelength of the $\mathrm{X}$-rays, the phase shift $\Phi$ can be calculated by two-dimensional integration (Morgan et al., 2012; Berujon, Ziegler et al., 2012). Simultaneously, transmission and dark-field images can also be obtained, from the changes in the intensity and the visibility of the speckle pattern induced by the sample, revealing information about absorption and scattering (Berujon, Wang et al., 2012).

As a demonstration of the potential applications in geological science, we selected one picrite (an important chemical end-member magma) sample from Iceland. It is composed of olivine macrocrystals set in a microcrystalline matrix that also contains some olivine. The thickness of the sample is $530 \mu \mathrm{m}$. Three-dimensional textural information is key to understanding cooling histories, and also to contextualize the chemical information of the materials. Attenuation contrast works well for distinguishing between crystals and air for porous samples. However, because of the chemical similarity between the macrocrystal and the matrix there is very little difference in attenuation, which means that phase contrast is highly valuable (Polacci $e t a l ., 2010)$. The development of this imaging system allows for new dynamic experiments to be designed that test long-held assumptions regarding the origin and evolution of these and other magmas. As shown in Fig. 5, the multimodal images of the picrite sample are retrieved using the speckle-based technique. The transmission image is shown in Fig. 5(e), and it exhibits poor absorption contrast
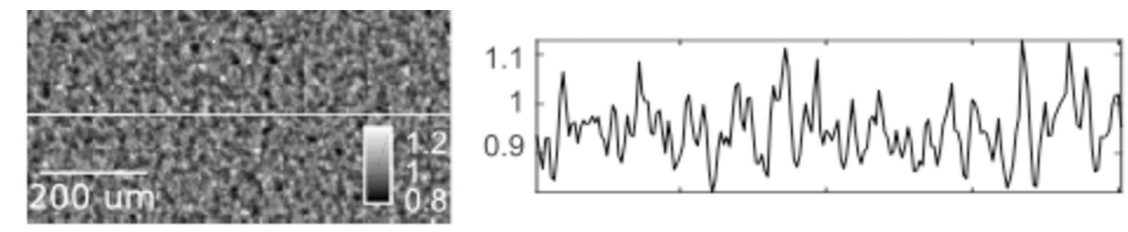

(a)

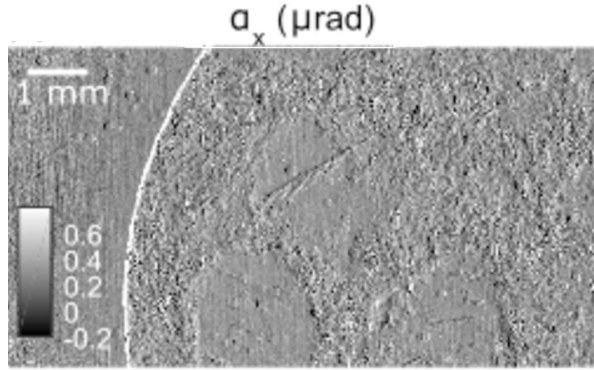

(b)

(d)

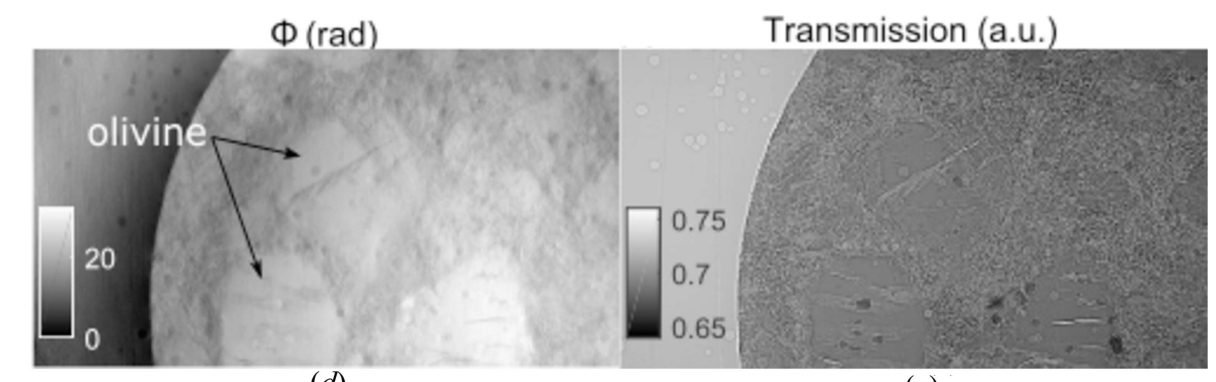

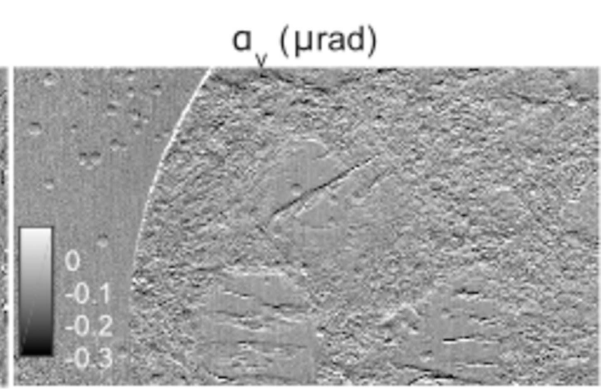

(c)
Figure 5

$(e)$

(a) Flat-field-corrected speckle image generated by the sandpaper and the profile taken along the line. $(b)$ Refraction angles in horizontal and $(c)$ in vertical directions, $(d)$ integrated phase, and (e) transmission image of a volcanic rock sample. 
between the olivine and the host groundmass. While this image can be interpreted by eye, it is difficult to segment digitally. The phase contrast image in Fig. $5(d)$, integrated from the horizontal and vertical refraction angles in Figs. 5(b) and $5(c)$, produces better contrast which can be segmented more easily. Further work is under way to apply this technique to tomographic imaging, where virtual slices can be reconstructed without the need for preparing thin sections of the sample. Combined with tomography, multimodal images will allow quantitative retrieval of the complex refractive indices of the materials in the sample, and can be applied for material characterization and quantitative analysis (Zanette, Zdora et al., 2015). Multimodal imaging also offers the extra scattering information that traditional attenuation imaging cannot. For example, it can be used to study water transportation across construction materials (Yang et al., 2015). With the development of the improved white-beam detecting system, such scientific applications are also possible at beamline B16. By enhancing the applicability and utility of BM beamlines for imaging, synchrotron X-ray users have expanded the opportunity to conduct a greater number of experiments, by providing an alternative to heavily over-subscribed insertiondevice-based imaging beamlines.

\section{Conclusion}

A white-beam imaging system has been designed, tested and established at the B16 beamline at Diamond. Continuous nitrogen flow in the space between the scintillator and the reflecting mirror has proved to be an effective way of preventing accumulation of dust particles on the scintillator, which would otherwise significantly degrade image quality over time. Similar concepts can be applied to other existing $\mathrm{X}$-ray imaging systems. The spatial resolution and FOV can be adjusted continuously by varying the positions of the lens and the camera with motorized stages, without the need to change to objectives with different magnifications. Demonstrations for fast tomography and multimodal phase-contrast imaging show that the X-ray imaging system developed will find wide applications, such as in the biomedical and geological sciences. With the further development of the detector, the white-beam imaging system will be routinely used, for example to study dynamic behavior of samples in fly-scan mode. The design of the imaging system is cost-efficient and flexible, and can be implemented easily on other synchrotron beamlines that provide intense X-ray flux.

\section{Acknowledgements}

This work was carried out with the support of the Diamond Light Source Ltd, UK. The authors wish to thank Matthew Pankhurst and Thor Thordarson for access to the geological samples and for analyzing the results. We would like to acknowledge Andrew Malandain and Hiten Patel for their technical assistance, and Ian Pape and Oliver Fox for their assistance during beam time at B16.

\section{References}

Agematsu, H., Abe, S., Shiozaki, K., Usami, A., Ogata, S., Suzuki, K., Soejima, M., Ohnishi, M., Nonami, K. \& Ide, Y. (2005). Bull. Tokyo Dent. Collect. 46, 7-15.

Agematsu, H., Watanabe, H., Yamamoto, H., Fukayama, M., Kanazawa, T. \& Miake, K. (1990). Bull. Tokyo Dent. Collect. 31, 163-173.

Baker, D. R., Brun, F., O'Shaughnessy, C., Mancini, L., Fife, J. L. \& Rivers, M. (2012). Nat. Commun. 3, 1135.

Berujon, S., Wang, H. \& Sawhney, K. (2012). Phys. Rev. A, 86, 063813.

Bérujon, S., Ziegler, E., Cerbino, R. \& Peverini, L. (2012). Phys. Rev. Lett. 108, 158102.

Bonse, U., Nusshardt, R., Busch, F., Pahl, R., Kinney, J. H., Johnson, Q. C., Saroyan, R. A. \& Nichols, M. C. (1991). J. Mater. Sci. 26, 4076-4085.

Diemoz, P. C., Bravin, A., Sztrókay-Gaul, A., Ruat, M., Grandl, S., Mayr, D., Auweter, S., Mittone, A., Brun, E., Ponchut, C., Reiser, M. F., Coan, P. \& Olivo, A. (2016). Phys. Med. Biol. 61, 87508761.

Eakins, D. E. \& Chapman, D. J. (2014). Rev. Sci. Instrum. 85, 123708.

Etoh, T. G., Poggemann, D., Kreider, G., Mutoh, H., Theuwissen, A. J. P., Ruckelshausen, A., Kondo, Y., Maruno, H., Takubo, K., Soya, H., Takehara, K., Okinaka, T. \& Takano, Y. (2003). IEEE Trans. Electron Devices, 50, 144-151.

Janda, R. (1995). Biomaterials, 16, 209-217.

Kalender, W. A., Seissler, W., Klotz, E. \& Vock, P. (1990). Radiology, 176, 181-183.

Koch, A., Raven, C., Spanne, P. \& Snigirev, A. (1998). J. Opt. Soc. Am. A, 15, 1940-1951.

Lak, M., Néraudeau, D., Nel, A., Cloetens, P., Perrichot, V. \& Tafforeau, P. (2008). Microsc. Microanal. 14, 251-259.

Lee, J. S., Weon, B. M. \& Je, J. H. (2013). J. Phys. D, 46, 494006.

Luo, S. N., Jensen, B. J., Hooks, D. E., Fezzaa, K., Ramos, K. J., Yeager, J. D., Kwiatkowski, K. \& Shimada, T. (2012). Rev. Sci. Instrum. 83, 073903.

Marton, Z., Nagarkar, V. V., Miller, S. R., Brecher, C., Bhandari, H. B., Kenesei, P., Ross, S. K., Almer, J. D. \& Singh, B. (2014). J. Phys. Conf. Ser. 493, 012017.

Modregger, P., Cremona, T. P., Benarafa, C., Schittny, J. C., Olivo, A. \& Endrizzi, M. (2016). Sci. Rep. 6, 30940.

Momose, A., Yashiro, W., Maikusa, H. \& Takeda, Y. (2009). Opt. Express, 17, 12540-12545.

Morgan, K. S., Paganin, D. M. \& Siu, K. K. W. (2012). Appl. Phys. Lett. 100, 124102-124104.

Olbinado, M. P., Just, X., Gelet, J.-L., Lhuissier, P., Scheel, M., Vagovic, P., Sato, T., Graceffa, R., Schulz, J., Mancuso, A., Morse, J. \& Rack, A. (2017). Opt. Express, 25, 13857-13871.

Pagot, E., Cloetens, P., Fiedler, S., Bravin, A., Coan, P., Baruchel, J., Härtwig, J. \& Thomlinson, W. (2003). Appl. Phys. Lett. 82, 34213423.

Pfeiffer, F., Bech, M., Bunk, O., Kraft, P., Eikenberry, E. F., Brönnimann, C., Grünzweig, C. \& David, C. (2008). Nat. Mater. 7, 134-137.

Pidol, L., Kahn-Harari, A., Viana, B., Virey, E., Ferrand, B., Dorenbos, P., de Haas, J. T. M. \& van Eijk, C. W. E. (2004). IEEE Trans. Nucl. Sci. 51, 1084-1087.

Polacci, M., Mancini, L. \& Baker, D. R. (2010). J. Synchrotron Rad. 17, 215-221.

Rack, A., Weitkamp, T., Riotte, M., Grigoriev, D., Rack, T., Helfen, L., Baumbach, T., Dietsch, R., Holz, T., Krämer, M., Siewert, F., Meduňa, M., Cloetens, P. \& Ziegler, E. (2010). J. Synchrotron Rad. 17, 496-510.

Sanchez del Rio, M. \& Dejus, R. J. (2004). Proc. SPIE, 5536, 171-174.

Sawhney, K. J. S., Dolbnya, I. P., Tiwari, M. K., Alianelli, L., Scott, S. M., Preece, G. M., Pedersen, U. K., Walton, R. D., Garrett, R., Gentle, I., Nugent, K. \& Wilkins, S. (2010). AIP Conf. Proc. 1234, 387-390. 
Spanne, P., Raven, C., Snigireva, I. \& Snigirev, A. (1999). Phys. Med. Biol. 44, 741-749.

Stampanoni, M., Borchert, G., Wyss, P., Abela, R., Patterson, B., Hunt, S., Vermeulen, D. \& Rüegsegger, P. (2002). Nucl. Instrum. Methods Phys. Res. A, 491, 291-301.

Sumikawa, D. A., Marshall, G. W., Gee, L. \& Marshall, S. J. (1999). Pediatr. Dent. 21, 439-444.

Walker, S. M., Schwyn, D. A., Mokso, R., Wicklein, M., Müller, T., Doube, M., Stampanoni, M., Krapp, H. G. \& Taylor, G. K. (2014). PLoS Biol. 12, e1001823.

Wang, H., Kashyap, Y., Cai, B. \& Sawhney, K. (2016). Sci. Rep. 6, 30581.

Wang, H., Kashyap, Y. \& Sawhney, K. (2015). Opt. Express, 23, 23310-23317.

Wang, H., Kashyap, Y. \& Sawhney, K. (2016). Sci. Rep. 6, 20476.
Wilkins, S. W., Gureyev, T. E., Gao, D., Pogany, A. \& Stevenson, A. W. (1996). Nature (London), 384, 335-338.

Willner, M., Bech, M., Herzen, J., Zanette, I., Hahn, D., Kenntner, J., Mohr, J., Rack, A., Weitkamp, T. \& Pfeiffer, F. (2013). Opt. Express, 21, 4155-4166.

Workman, J., Cobble, J., Flippo, K., Gautier, D. C., Montgomery, D. S. \& Offermann, D. T. (2010). Rev. Sci. Instrum. 81, $10 \mathrm{E} 520$.

Yang, F., Griffa, M., Bonnin, A., Mokso, R. C. D. I. B., DI Bella, C., Münch, B., Kaufmann, R. \& Lura, P. (2015). J. Microsc. 261, 88-104.

Zanette, I., Enders, B., Dierolf, M., Thibault, P., Gradl, R., Diaz, A., Guizar-Sicairos, M., Menzel, A., Pfeiffer, F. \& Zaslansky, P. (2015). Sci. Rep. 5, 9210.

Zanette, I., Zdora, M.-C., Zhou, T., Burvall, A., Larsson, D. H., Thibault, P., Hertz, H. M. \& Pfeiffer, F. (2015). Proc. Natl Acad. Sci. USA, 112, 12569-12573. 